\title{
Procesos educativos sobre enfermedad de Chagas con escolares de nivel básico de escuelas públicas de México
}

\author{
Luz Arenas Monreal ${ }^{1}$, Lilian E. Pacheco Magaña ${ }^{1}$, Pastor Bonilla Fernández ${ }^{1}$, Celina M. Rueda Neria ${ }^{1}$, \\ José Francisco Flores Alatorre ${ }^{2}$, Cristina Raquel Caballero García ${ }^{2}$ \\ ${ }^{1}$ Instituto Nacional de Salud Pública. Centro de Investigaciones en Sistemas de Salud. México \\ ${ }^{2}$ Facultad de Odontología. Universidad Central del Paraguay. Asunción, Paraguay
}

Cómo referenciar este artículo/ How to reference this article:

\begin{abstract}
Arenas Monreal L, Pacheco Magaña LE, Bonilla Fernández P, Rueda Neria CM, Flores Alatorre JF, Caballero García CR. Procesos educativos sobre enfermedad de Chagas con escolares de nivel básico de escuelas públicas de México. Mem. Inst. Investig. Cienc. Salud. 2018; 16(1): 14-18
\end{abstract}

\section{R E S U M E N}

Se presentan los cambios en conocimientos y prácticas sobre el vector y la enfermedad de Chagas con escolares de México posterior a una intervención educativa. La identificación del vector aumentó posterior a las sesiones educativas (pre $=36,15 \%$ vs post $=79,30 \%$ ). Aumentaron los conocimientos acerca de la enfermedad de Chagas (30,90\% vs 64,72\%, valor de $p<0,0001$ ) y de las acciones preventivas para evitar que el vector habite dentro de sus entornos $(14,29 \%$ vs $61,81 \%$, valor de $p<0,0001)$. Los escolares pueden jugar un papel fundamental en la prevención de esta problemática.

Palabras clave: escolares, Tripanosoma cruzi, enfermedad de Chagas.

\section{Educational processes on Chagas disease in schoolchildren from public schools in Mexico}

\section{A B S T R A C T}

This communication presents the changes in knowledge and practices about Chagas disease and its insect-vector, with Mexican schoolchildren after an educational intervention. Vector identification increased after the educational sessions ( $p r e=36.15 \% \%$ vs post $=79.30 \%$ ). Not only the knowledge about Chagas disease increased $(30.90 \%$ vs. $64.72 \%$, p value $<0.0001$ ) but also about preventive actions to avoid the vector dwelling in their homes and schools $(14.29 \%$ vs. $61.81 \%$, p value $<0.0001)$. Schoolchildren can play a vital role in the prevention of this disease.

Keywords: schoolchildren, Trypanosoma cruzi, Chagas disease.

\section{INTRODUCCIÓN}

La enfermedad de Chagas (ECh) fue descrita por primera vez por Carlos Chagas en 1.909 en Brasil. Es causada por un protozoo, Trypanosoma Cruzi, el cual es transmitido a los humanos principalmente a través de un insecto vector: triatóminos de la familia Reduviidae, orden Hemiptera (chinches), Subfamilia Triatominae. Otros modos de transmisión son: transfusional, congénito, trasplante de órganos y oral ${ }^{(1)}$.

La (ECh) es endémica en 21 países de América Latina ${ }^{(2,3)}$. Desde 1990, los países afectados por la ECh, en especial donde esta enfermedad es endémica, se organizaron con el apoyo de la Organización Panamericana de la Salud / Organización Mundial de la Salud y se ejecutaron iniciativas subregionales de prevención y control para esta enfermedad. Estas iniciativas se efectuaron por regiones: Cono Sur (1992), América Central (1997), países andinos (1998), países amazónicos (2003) y México (2004), este último país, desde 2013 se integró con los países de Centroamérica en la Iniciativa de Centroamérica y México. A 
través de estas iniciativas se han obtenido avances, tales como la implantación del tamizaje universal de donantes de sangre entre otros aspectos. Las iniciativas de las Américas han permitido alcanzar reducciones importantes del número de casos agudos y de la presencia intradomiciliaria de vectores triatominos en todas las zonas endémicas. El número estimado de personas infectadas pasó de 30 millones en 1990 a 6 a 8 millones en el 2010. En esos 20 años, la incidencia anual decreció de 700.000 a 28.000 casos $^{(4)}$.

Ramsey et al, 2015, señalan que en las últimas estimaciones, la prevalencia de esta enfermedad está disminuyendo en la región de América del Sur, sin embargo, la mayoría de estos países no tienen vigilancia epidemiológica activa ${ }^{(5)}$. La carga anual en costos por la atención en salud, se calcula en 627,46 millones de dólares y en 806.170 años de vida ajustados por discapacidad ${ }^{(6)}$.

La (ECh) se clasifica dentro de las enfermedades tropicales desatendidas (ETD). Hotez et al, 2008, reportan que en América Latina las ETD se concentran geográficamente en 11 diferentes subregiones en las que coexisten características sociodemográficas (alta marginación, pobreza, vivienda deficiente, escasos o nulos servicios de saneamiento básico y acceso a servicios de salud) con aspectos ambientales únicos como las áreas del altiplano seco y frío, el chaco seco y estéril (Argentina, Bolivia, Paraguay), montañas aisladas de México, Centroamérica y la Amazonía, así como cuencas del caribe. Estos autores señalan que en estas áreas la ECh continúa siendo altamente endémica, más que cualquier otra $\operatorname{ETD}^{(7)}$.

Actualmente migrantes provenientes de países con alta prevalencia en América Latina de ECh, son la causa de que existan casos de esta enfermedad en América del Norte, Europa y Asía ${ }^{(5,8)}$.

La Organización Mundial de la Salud planteó objetivos para el control y la eliminación de la enfermedad de Chagas con base en dos pilares: 1) interrupción de la transmisión (vectorial intradomiciliar, reducir el riesgo de transmisión oral, interrumpir la transmisión adquirida a través de transfusiones de sangre y trasplante de órganos, mejora del saneamiento básico y uso de mosquiteros) y 2) atención a los pacientes ${ }^{(9)}$.

En México, se reportan 71 millones de habitantes en riesgo directo. La prevalencia no se conoce con exactitud pero se estima que está entre $0,013 \%$ y $3,12 \%{ }^{(3)}$.

Actualmente, en el abordaje de esta enfermedad se señala la necesidad de incorporar promoción y educación para la salud, así como la participación de la población ${ }^{(10,11)}$.

El objetivo de este estudio fue incrementar los conocimientos y prácticas preventivas sobre la enfermedad de Chagas en escolares.

\section{MATERIAL Y MÉTODO}

Estudio cuasi-experimental con mediciones pre/post-intervención educativa con 343 escolares de primaria $\left(4^{\circ}, 5^{\circ}, 6^{\circ}\right.$ grados) y secundaria $\left(1^{\circ}, 2^{\circ}, 3^{\circ}\right.$ grados), ciclo escolar 2011-2012, en cuatro escuelas públicas del estado de Morelos de zona urbana y rural. El estudio fue aprobado por las Comisiones de Investigación y Ética del Instituto Nacional de Salud Pública.

Se aplicó un cuestionario de conocimientos. La primera pregunta indagaba el conocimiento sobre el vector, a quienes respondían positivamente se les mostraba una tarjeta con trece imágenes (cada imagen con número), de los cuales tres de ellas $(1,7$ y 13$)$ correspondían a chinches hematófagas y 10 a insectos que depredan a otros insectos. La tarjeta fue diseñada por la Dra. Janine M. Ramsey, quien ha utilizado la tarjeta en múltiples estudios en diversas regiones de México. La Dra Ramsey capacitó a integrantes del equipo en el uso e interpretación de la tarjeta. La utilización de esta tarjeta permitió identificar aquellas personas que habían tenido experiencia directa con el vector y su probable presencia en su entorno.

Se efectuaron 10 sesiones educativas con metodología participativa, las cuales fueron coordinadas por facilitadoras educativas previamente capacitadas en la temática. Las sesiones se enfocaron en la identificación del vector, los sitios en los que habita, características del piquete, sintomatología de la enfermedad de Chagas y acciones preventivas. Se efectuaron actividades como: periódico mural, recorridos comunitarios para identificar sitios con riesgo, radio escolar y carteles para difundir información de esta problemática tanto dentro como fuera de la escuela.

La información fue capturada y analizada utilizando el paquete estadístico Stata v.12. Se obtuvieron frecuencias simples y diferencia de proporciones entre la primera y segunda 
medición con un nivel de confianza del 95\%. El Valor Predictivo Positivo (VPP) de identificación del vector se obtuvo a partir de la proporción resultante de los escolares que dijeron conocer el vector y lo identificaron correctamente en la tarjeta, entre el total de escolares que respondieron conocerlo.

\section{RESULTADOS}

En la comunidad urbana el $73,9 \%$ de las viviendas presentaban techo de concreto, paredes de block $(65,45 \%)$ y piso de cemento $(70,33 \%)$, con presencia de animales domésticos (78,86\%). El $67,5 \%$ de las viviendas de zona rural tenían techo de lámina, paredes de ladrillo (41,25\%), el $17,5 \%$ con piso de tierra, y con animales domésticos el $86,25 \%$.

Participaron 158 niñas (edad promedio 12,51 años, DE: 1,94) y 182 niños (edad promedio 12,61 años, DE: 1,88). Tres escolares no reportaron su edad.

El Valor Predictivo Positivo aumentó posterior a las sesiones educativas (VPP Preintervención $=58,07 \%$, VPP Post-intervención $=89,71 \%$ ) (Figura 1). Se encontró aumento en el conocimiento de la enfermedad de Chagas (Pre-intervención 30,90\% Vs Post-intervención $64,72 \%$, diferencia: $33,82 \%$, valor de $p=0,0000$, IC: -.4085388 .267846 ) y de las acciones preventivas (Pre-intervención 14,29\% Vs Post-intervención 61,81\%, diferencia: 47,52\%, valor de $p=0,0000$, IC: $-.5385837-.4118536)$.

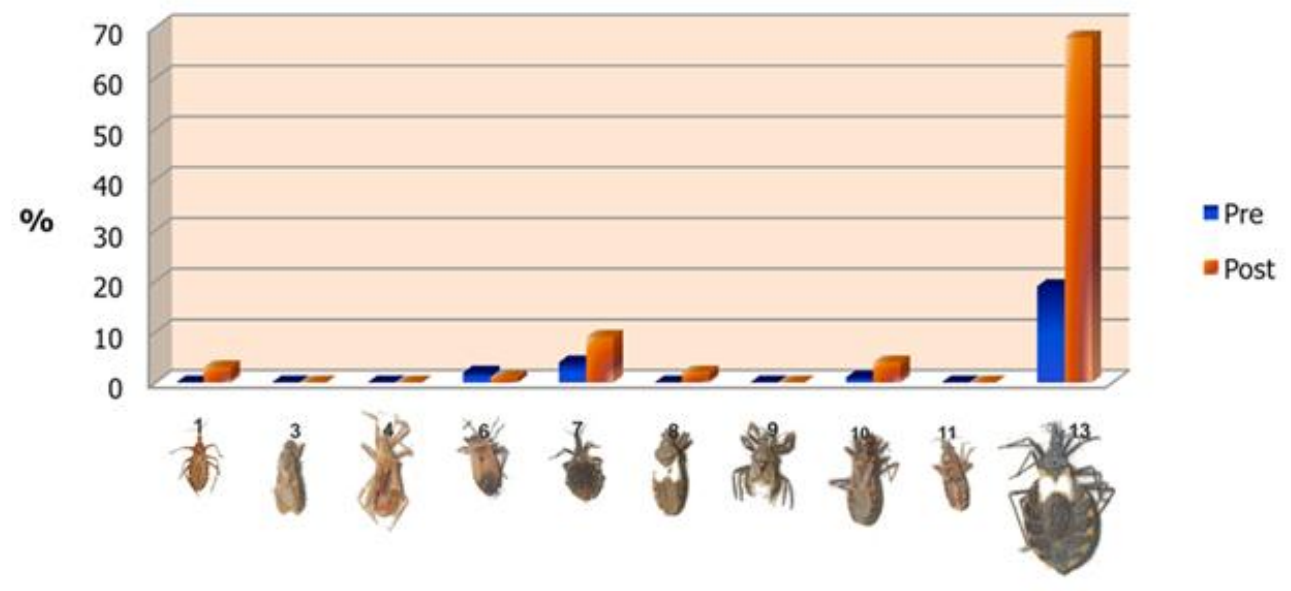

Figura 1. Identificación de la chinche de chagas por escolares. $n=343$

Hubo diferencias estadísticamente significativas por sexo en el conocimiento e identificación del vector en la fase pre-intervención (diferencia $=22,22 \%$, valor de $\mathrm{p}=0,0038$, IC:-.3814686 -.0629758), conservándose la diferencia post-intervención (diferencia $=9,02 \%$, valor de $p=0,0232$, IC:-.178526 -.0018019), este conocimiento fue adquirido en mayor porcentaje por hombres. En las diferencias al interior de cada grupo (hombres vs mujeres) post-intervención se registró un aumento estadísticamente significativo del $50 \%$ en promedio.

Se incrementó el conocimiento sobre los sitios en los que suele habitar el vector (lugares escondidos, oscuros y secos) y de las acciones que se pueden realizar para prevenir la presencia del insecto en la vivienda y sus alrededores. Entre estas actividades destacan: limpiar casa y patio $(49,3$ vs. $77,3 \%)$, limpieza $(28,3$ vs $33,5 \%)$ y mover los muebles ( 21 a $33,2 \%)$. Disminuyó el porcentaje de quienes consideran la fumigación como el principal recurso para combatir el vector. Hubo significancia estadística $(p<0.05$, nivel de confianza $95 \%$ ) en las diferencias de proporción de limpiar casa/ patio y fumigar en la medición pre y post intervención.

En cada escuela se formó un comité de escolares para promover acciones de abogacía y para efectuar medidas preventivas tanto en la escuela como en la comunidad.

\section{DISCUSIÓN}

Este estudio demostró que a través de una intervención educativa en escolares es posible incrementar los conocimientos y su participación en acciones preventivas. 
Los resultados reportados en otros estudios son semejantes a nuestros hallazgos. En una investigación efectuada en Yucatán, se señaló que los escolares son actores sociales fundamentales para estrategias de movilización poblacional en contra de los triatomas y la $\mathrm{ECh}^{(10)}$. Otra intervención educativa con escolares, efectuada de igual forma en Yucatán reportó un incremento de conocimiento en los escolares, quienes además participaron en la recolección de vectores y en difundir información con sus familias y comunidad ${ }^{(12)}$. Crocco $L$ et al., 2005, señalan la importancia de la participación de escolares con previa capacitación, no sólo en la vigilancia sino en caracterizar viviendas en riesgo e identificar el vector ${ }^{(11)}$. Al contrario de nuestro estudio, Ferrero et al., 2015, en una intervención educativa con escolares en Argentina no encontraron cambios en los conocimientos ${ }^{(13)}$.

Otros estudios indagan los conocimientos que tienen los escolares pero sin efectuar intervención educativa. Estos estudios reportan que en general los escolares conocen al vector pero tienen saberes limitados en cuanto a medidas preventivas ${ }^{(14,15)}$. Nuestro estudio es diferente ya que la intervención educativa permitió el incremento de conocimientos y la participación en acciones preventivas.

Las acciones educativas y la difusión creativa de información ${ }^{(16)}$, permiten la alfabetización sanitaria en una problemática en la que la población y los escolares no están familiarizados en la importancia que tiene la vivienda y el entorno en la presentación de esta enfermedad. La alfabetización sanitaria en la ECh puede contribuir para que la población participe activamente en el control este problema. Diversos autores señalan que la participación comunitaria y el dialogo con la población civil, es un componente fundamental en conjunto con otra serie de medidas, para la eliminación efectiva del vector $^{(17,18)}$.

El trabajo con escolares y aún más con la comunidad escolar de los planteles educativos es un espacio crucial para incrementar la alfabetización sanitaria sobre la ECh, lo cual puede contribuir para que se efectúen acciones dentro de los espacios comunitarios para el control y prevención del vector y la enfermedad.

\section{Agradecimientos}

A los escolares, profesores y comunidades. A la Dra. Janine M. Ramsey por compartir la tarjeta utilizada en este estudio. A Ernesto Pacheco por su apoyo con la figura incluida en este manuscrito. El proyecto fue coordinado por el Instituto Nacional de Salud Pública de México (INSP), con financiamiento del Consejo Nacional de Ciencia y Tecnología (CONACYT) Proyecto $N^{\circ} 114696$.

\section{Declaración de conflicto de intereses}

Los autores declaran no tener conflicto de intereses.

\section{REFERENCIAS BIBLIOGRAFICAS}

1. Moncayo A, Silveira AC. Current epidemiological trends for Chagas disease in Latin America and future challenges in epidemiology, surveillance and health policy. Mem Inst Oswaldo Cruz 2009; 104(SupplI): 17-30.

2. Clayton J. Chagas disease. Nature. 2010; 465(7301): S4-S5.

3. Ramsey JM, Elizondo-Cano M, SanchezGonzález G, Peña-Nieves A, Figueroa-Lara A. Opportunity Cost for early treatment of Chagas disease in Mexico. PLoS Negl Trop Dis. 2014; 4: e2776.

4. Organización Panamericana de la Salud. Información general: Enfermedad de Chagas. Disponible en: http://www.paho.org/hq/index.php?option= com_content\&view $=$ article\&id $=5856 \% 3$ A201 1-informacion-general-enfermedadchagas\&catid=3591\%3Achagasdisease\&Itemid=0\&lang=es

5. Ramsey JM, Townsend Peterson A, Carmona-Castro O, Moo-Llanes DA,
Nakazawa $Y$, Butrick $M$, et al. Atlas of Mexican Triatominae (Reduviidae: Hemiptera) and vector transmission of Chagas disease. Mem Inst Oswaldo Cruz 2015; 110(3): 339-52.

6. Lee BY, Bacon KM, Bottazzi ME, Hotez PJ. Global economic burden of Chagas disease: a computational simulation model. Lancet Infect Dis 2013; 13(4): 342-8.

7. Hotez PJ, Bottazzi ME, Franco-Paredes C, Ault SK, Periago MR. The neglected tropical diseases of Latin America and the Caribbean: A review of disease burden and distribution and a roadmap for control and elimination. PLoS Negl Trop Dis 2008; 2(9): e300.

8. Pinto-Dias JC. Tendencias sociales de la enfermedad de Chagas para las próximas décadas. Salud Colectiva. 2012; 8(Supl 1): S39-S48.

9. Organización Mundial de la Salud. La enfermedad de Chagas (tripanosomiasis americana). Disponible en: 
http://www.who.int/mediacentre/factsheets/ fs340/es/

10. Waleckx E, Camara-Mejia J, Ramírez-Sierra $M$, Cruz-Chan V, Rosado-Vallado $M$, Vazquez-Narvaez $S$, et al. An innovative ecohealth intervention for Chagas disease vector control in Yucatan, Mexico. Trans $\mathrm{R}$ Soc Trop Med Hyg. 2015; 109: 143-9.

11. Crocco L, Rodríguez C, Catalá S, Nattero J. Enfermedad de Chagas en Argentina: herramientas para que los escolares vigilen y determinen la presencia de factores de riesgo en sus viviendas. Cad Saúde Pública. 2005; 21(2): 646-51.

12. Polanco-Rodríguez AG, Ruiz-Piña $H A$, Puerto FI. La investigación participativa en niños como herramienta en la promoción de la salud para la prevención de la Enfermedad de Chagas en Yucatán, México. Revista Biomédica. 2017; 28(3): 123-35.

13. Ferrero ME, Meinardi $E$, Cecere $C$. Educación para la salud en áreas rurales. Enfermedad de Chagas en Tucumán, Argentina. Revista Iberoamericana de Educación. 2015; 69(2): 89-110.

14. Villela MM, Pimenta DN, Lamounier PA, Dias JCP. Evaluation of knowledge and practices related to Chagas disease and its vectors among adults and children in an endemic region in Minas Gerais State, Brazil. Cad. Saúde Pública. 2009; 25(8): 1701-10.

15. Cabrera R, Mayo C, Suárez N, Infante C, Náquira C, García-Zapata MTA. Conocimientos, actitudes y prácticas sobre la enfermedad de Chagas en población escolar de una zona endémica del Perú. Cad. Saúde Pública. 2003; 19(1): 147-54.

16. Sanmartino M, Mengascini A, Menegaz A, Mordeglia C, Ceccarelli S. Miradas caleidoscópicas sobre el Chagas. Una experiencia educativa en el museo de La Plata. Revista Eureka sobre Enseñanza y Divulgación de las Ciencias. 2012; 9(2): 265-73.

17. Abad-Franch F, Vega MC, Rolón MS, Santos WS, Rojas de Arias A. Community participation in Chagas disease vector surveillance: Systematic review. PLoS Negl Trop Dis 2011; 5(6): e1207.

18. Tarleton RL, Gürtler RE, Urbina JA, Ramsey J, Viotti R. Chagas disease and the London declaration on neglected tropical diseases. PLoS Negl Trop Dis. 2014; 8(10): e3219. 